\title{
An efficient FE model and LSE method for accurate calculation of transverse stresses in laminated sandwich plates
}

\author{
R. P. Khandelwal, A. Chakrabarti \& P. Bhargava \\ Department of Civil Engineering, Indian Institute of Technology, India
}

\begin{abstract}
Accurate evaluation of transverse stresses in laminated composites and sandwich plates using 2D FE models involves cumbersome post processing techniques. In this paper a simple and efficient method has been proposed for accurate evaluation of through-the-thickness distribution of transverse stresses in composites and sandwich laminates by using a displacement based $\mathrm{C}^{0} \mathrm{FE}$ model derived from refined higher order shear deformation theory (RHSDT) and a Least Square Error (LSE) method. The $\mathrm{C}^{0} \mathrm{FE}$ model satisfies the inter-laminar shear stress continuity conditions at the layer interfaces and zero transverse shear stress conditions at the top and bottom of the plate. In this model the first derivatives of transverse displacement have been treated as independent variables to circumvent the problem of $\mathrm{C}^{1}$ continuity associated with the above plate theory (RHSDT). The LSE method is applied to the 3D equilibrium equations of the plate problem at the post-processing stage, after in-plane stresses are calculated by using the above FE model based on RHSDT. Thus the proposed method is quite simple and elegant compared to the usual method of integrating the 3D equilibrium equations at the post-processing stage for the calculation of transverse stresses in a composite laminate. Accuracy of the proposed method is demonstrated in the numerical examples through comparison of the present results with those obtained from different models based on higher order shear deformation theory (HSDT) and 3D elasticity solutions.

Keywords: composite sandwich laminates, $C^{0} R H S D T$, transverse shear stresses, LSE method.
\end{abstract}




\section{Introduction}

Composites and sandwich structural components are widely used in mechanical, aerospace, civil, ocean and other engineering fields due to their advantage of high stiffness and strength to weight ratio. However, these structures are weak in shear due to their low shear modulus compared to extensional rigidity. Thus the effect of shear deformation is quite significant which may lead to failure. However, the accurate evaluation of transverse shear stresses is very difficult using 2D models available in the literature. To predict accurate transverse shear stresses within the framework of 2D analysis extensive research is going on over the years for developing appropriate and accurate models.

The widely used 2D displacement-based plate theories can be categorized into two groups and they are (1) equivalent single-layer plate theory (ESLT) and (2) layer-wise plate theory (LWT). In equivalent single-layer theory [1-7] the deformation of the plate is expressed in terms of unknown parameters of a single plane, which is usually taken as the middle plane of the plate. These are similar to Reissner-Mindlin's plate theory (i.e., the first-order shear deformation theory, FSDT) which requires shear correction factor but there are some improvements, which allow the warping of plate sections to have a higher-order variation of transverse shear stresses/strains along the thickness. In the layer wise theory [8-11] the deformation of the plate is expressed in terms of unknowns of a number of planes, which are taken at the layer interfaces and also at some intermediate levels in some cases. The mathematical involvement in these plate theories is quite heavy and the solution becomes quite expensive in a multilayered plate, as the unknowns are dependent on number of layers. There is another class of layer wise plate theories [12-18] where the unknowns of different planes are expressed in terms of those of a particular plane using the condition of shear stress continuity at the layer interfaces and the number of unknowns is dramatically reduced.

In this context, the first-order shear deformation theory (Yang et al. [1]) maybe considered as the simplest option where an arbitrary shear correction factor is used since the transverse shear strain is assumed to have uniform variation over the entire plate thickness. The first order shear deformation theory, which assumes a constant transverse shear strain across the thickness direction and a shear correction factor, is introduced to correct the discrepancy between the actual transverse shear stress distribution and those assumed in this theory. The performance of first-order shear deformation theory is dependent on shear correction factors (Vlachoutsis [2]). For a better representation of the transverse shear deformations, higher order plate theories (HSDT) are proposed by Lo et al. [3], Reddy [4], Manjunatha and Kant [5], and a few others, in which the use of shear correction factor could be eliminated. It gives continuous variation of transverse shear strain across the entire thickness, which leads to discontinuity in the variation of the transverse shear stresses at the layer interfaces. But the actual behavior of laminated plate is the opposite i.e., the transverse shear stress is continuous at the interfaces whereas the strains may be discontinuous. 
In order to overcome the above problem, Srinivas [8], Toledano and Murakami [9], Robbins and Reddy [10], Li and Liu [11], and some other investigators proposed layer-wise plate theories taking unknowns at each layer interface. These plate theories perform well but they require significant computational involvement in analyzing a multi-layered plate since the number of unknowns increases with the number of layers. A major development in this direction is due to Di Sciuva [12], Murakami [13], Liu and Li [14], and few others. They proposed zigzag plate theory where layer-wise theory is initially used to represent the in-plane displacements having piecewise linear variation across the thickness. The unknowns at the different interfaces are subsequently expressed in terms of those at the reference plane through satisfaction of transverse shear stress continuity at the layer interfaces. A further improvement in this direction is due to Bhaskar and Varadan [15], Di Sciuva [16], Cho and Parmerter $[17,18]$ and some other investigators who considered the variation of in-plane displacements to be a superposition of a piecewise linearly varying field on an overall higher order variation. Carrera [19] and Demasi [20] considered higher order terms in the displacement field, using Murakami’s [21] zig-zag function and assumptions for transverse stresses brings about a large number of solution variables. However, applying static condensation technique allows us to eliminate the unknowns related to the transverse stresses and thus, to derive efficient plate theories (Demasi [22, 23]). Kapuria et al. [24], and Kapuria and Achary [25] have presented zigzag theory for hybrid beams and plates in which number of variable are reduced to FSDT by satisfying interface and boundary conditions, it yield approximately accurate results for cross ply only. Zhen and Wanji [26] proposed $\mathrm{C}^{0}$ type higher-order theory for bending analysis of laminated composite and sandwich plates. Zhen et al. [27] also proposed $\mathrm{C}^{0}$ type finite element based higher-order theory for accurately predicting natural frequencies of sandwich plate with soft core. These theories are usually referred as refined higher order shear deformation theory (RHSDT). However, there are very few $\mathrm{C}^{0}$ elements reported in the literature which can model the RHSDT.

Conventionally, the transverse stress recovery from the 3D equilibrium equations is dependent on the cumbersome integration of these equations through the thickness of laminate. Recently, Bhar and Satsangi [28] have proposed an accurate transverse stress evaluation in composite/sandwich thick laminates using $\mathrm{C}^{0} \mathrm{HSDT}$ and a novel post-processing technique. In this paper a simple least square of error (LSE) method is proposed to accurately calculate the transverse shear stresses. This method depends on an assumed variation of transverse stress field, suitably chosen and applied after the global analysis. This technique gives quite good results for transverse shear stresses compared to those obtained by using constitutive relations. However, the displacement formulation chosen in this paper is based on HSDT which gives continuous transverse shear strain variation across the thickness with possible discontinuity in the corresponding stresses. However, the shear stress field and the shear stress continuity conditions at the layer interfaces used in their formulation are not consistent with the corresponding strain/stress fields used in HSDT. As such there is further scope to improve the results for displacement and also getting 
accurate values of transverse shear stresses, if the formulation is based on RHSDT. In this connection it is to be noted that the displacement field chosen in the formulation of RHSDT is perfectly consistent with the stress field chosen along with the condition of stress continuity at the layer interfaces required in the formulation of LSE to calculate the transverse shear stresses accurately.

Considering all these aspects in view, an attempt has been made in this study to develop an improved FE plate model based on RHSDT to accurately predict the deflections and stresses of composites and sandwich laminates due to different loadings, boundary and geometric conditions. The nine noded $\mathrm{C}^{0}$ element proposed by Shankara and Iyengar [7] for simple higher order theory (HSDT) is upgraded to model the RHSDT in the present study. In the present paper the global response (i.e. displacement) is calculated first, using this efficient $\mathrm{C}^{0}$ FE model based on RHSDT and then an accurate prediction of transverse stresses is calculated from the $3 \mathrm{D}$ equilibrium equations using an efficient LSE method. It is interesting to note that in the proposed model, the displacement field used for calculation of unknown displacements and in-plane stresses are consistent with the stress field chosen along with the condition of stress continuity at the layer interfaces for calculation of transverse shear stresses based on the LSE method. The accuracy of the proposed finite element model is established by comparing the results with three dimensional elasticity and other finite element solutions.

\section{Formulation}

\subsection{FE model for displacements and in-plane stresses evaluation}

The in-plane displacement fields (fig. 1) are typical to those of RHSDT and are as below:

$$
u_{\alpha}=u_{\alpha}^{0}+\sum_{k=0}^{n u-1} S_{\alpha}^{k}\left(z-z_{k}\right) H\left(z-z_{k}\right)+\sum_{k=0}^{n l-1} T_{\alpha}^{k}\left(z-\rho_{k}\right) H\left(-z+\rho_{k}\right)+\xi_{\alpha} z^{2}+\varphi_{\alpha} z^{3}
$$

and

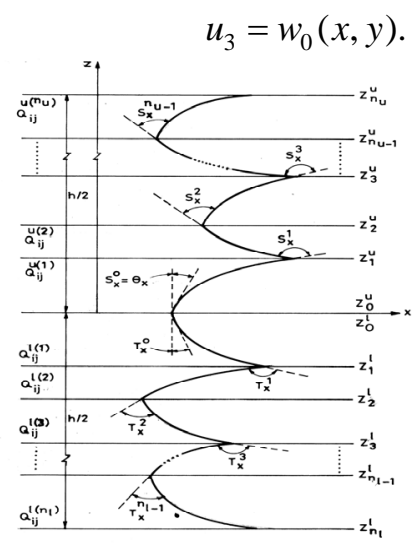

Figure 1: The displacement configuration for general lamination lay-up. 
The stress-strain relationship of a lamina (say, $k$-th) lamina having any fiber orientation with respect to structural axes system $(x-y-z)$ may be expressed as

$$
\{\bar{\sigma}\}=\left[\bar{Q}_{k}\right]\{\bar{\varepsilon}\} \text {. }
$$

By using minimizing the potential energy approach with respect to $\{\delta\}$, one can obtain

$$
[K]\{\delta\}=\{P\}
$$

where $[K]$ is the element stiffness matrix and $\{P\}$ the nodal load vector.

The details about FE Model are given in Singh et al. [33].

\subsection{The least square of error (LSE) method for transverse stress evaluation}

The 3D equilibrium equations in the absence of external and body forces may be written as below,

$$
\begin{aligned}
& \sigma_{x x, x}+\tau_{x y, y}+\tau_{x z, z}=0 \\
& \tau_{x y, x}+\sigma_{y y, y}+\tau_{y z, z}=0 \\
& \tau_{x z, x}+\tau_{y z, y}+\sigma_{z z, z}=0
\end{aligned}
$$

with usual conventions for the derivatives.

It is noted that the through-the-thickness variation of transverse shear stresses at an arbitrary point $\mathrm{P}(x, y)$ in the plan of the plate in thick laminates actually show nonlinear variation. As such the transverse shear stress component $\tau_{\mathrm{xz}}$ can be assumed to be distributed in a polynomial form of suitable order through the thickness of the $p$-th lamina at the point $\mathrm{P}(x, y)$. In the proposed study, this variation is assumed to be quadratic and is defined as below,

$$
\tau_{x z}^{(p)}=\alpha_{p 0}+z \alpha_{p 1}+z^{2} \alpha_{p 2}=\left[\begin{array}{lll}
1 & z & z^{2}
\end{array}\right]\left\{\alpha_{p}\right\}
$$

with $\alpha_{p i}(i=0,1,2)$ being unknown coefficients. It should be noted that this form is also consistent with the quadratic through-the-thickness variation of transverse shear stresses along with the condition of stress continuity at the layer interfaces derived from the displacement assumptions in eqn (1) based on the proposed RHSDT model. Denoting $\tau_{x z}^{(p b)}, Q_{x z}^{(p b)}$ and $\tau_{x z}^{(p t)}$ the shear stress value at the bottom, the resultant transverse shear force and the shear stress value at the top of the $p$-th layer respectively, one can obtain the following by using eqn (6)

$$
\left\{\tau_{x z}\right\}^{(p)}=\left\{\begin{array}{l}
\tau_{x z}^{(p b)} \\
Q_{x z}^{(p)} \\
\tau_{x z}^{(p t)}
\end{array}\right\}=\left[\begin{array}{ccc}
1 & z_{p b} & \left(z_{p b}\right)^{2} \\
z_{p 1} & z_{p 2} & z_{p 3} \\
1 & z_{p t} & \left(z_{p t}\right)^{2}
\end{array}\right]\left\{\begin{array}{l}
\alpha_{p 0} \\
\alpha_{p 1} \\
\alpha_{p 2}
\end{array}\right\}=\left[z_{p}\right]\left\{\alpha_{p}\right\}
$$


where $Z_{p b}$ and $Z_{p t}$ are the $z$-coordinates of the bottom and top surfaces of the $p$-th layer and

$$
\begin{aligned}
Z_{p i} & =\frac{\left(z_{p t}\right)^{i}-\left(z_{p b}\right)^{i}}{i} ; i=1,2,3 \\
& =\left[z_{p}\right]\left\{\alpha_{p}\right\} .
\end{aligned}
$$

Substituting $\left\{\alpha_{p}\right\}=\left[z_{p}\right]^{-1}\left\{\tau_{\mathrm{xz}}\right\}^{(\mathrm{p})}=[A]\left\{\tau_{\mathrm{xz}}\right\}^{(\mathrm{p})}$ from eqn (9) into eqn (6) one can get,

$$
\tau_{x z}^{(p)}=\left[\begin{array}{lll}
1 & z & z^{2}
\end{array}\right][A]\left\{\tau_{x z}\right\}^{(p)}=[\varphi]\left\{\tau_{x z}\right\}^{(p)}
$$

where $[\varphi]=\left[\begin{array}{lll}\varphi_{1} & \varphi_{2} & \varphi_{3}\end{array}\right]$ denote a kind of shape functions of the thickness coordinate such that

$$
\begin{gathered}
\varphi_{i}=A_{1 i}+A_{2 i} z+A_{3 i} z^{2} \\
\varphi_{i, z}=A_{2 i}+2 A_{3 i} z
\end{gathered}
$$

$A_{i j}$ being the element on the $i^{\text {th }}$ row and $j^{\text {th }}$ column of the $[\mathrm{A}]$ matrix.

From eqn (19),

$$
\begin{gathered}
\tau_{x z}^{(p)}=\varphi_{1} \tau_{x z}^{(p b)}+\varphi_{2} Q_{x z}^{(p)}+\varphi_{3} \tau_{x z}^{(p t)} \\
\tau_{x z, z}^{(p)}=\varphi_{1, z} \tau_{x z}^{(p b)}+\varphi_{2, z} Q_{x z}^{(p)}+\varphi_{3, z} \tau_{x z}^{(p t)} .
\end{gathered}
$$

Now, since the distribution of $\tau_{x z}^{(p)}$ through the thickness of $p$-th layer is given by eqn (12), the total square of error in satisfying the first of the equilibrium equations (14), over the thickness of $p$-th layer, may be written as,

$$
E_{p}=\int_{z_{p b}}^{z_{p t}}\left[\left(\tau_{x z, z}^{(p)}\right)+\left(\sigma_{x x, X}^{(p)}+\tau_{x y, y}^{(p)}\right)\right]^{2} d z
$$

where the in-plane stresses in the right most parenthesis are calculated from relevant constitutive relations for $p$-th layer and interpolation of smoothed mid plane nodal strains of the element, after performing the global finite element analysis. $E_{p}$, in eqn (14) should be minimum with respect to the unknown ply quantities $\tau_{x z}^{(p b)}, Q_{x z}^{(p b)}$ and $\tau_{x z}^{(p t)}$, as par the principle of the least square of errors. Hence,

$$
\frac{\partial E_{p}}{\partial \tau_{x z}^{(p b)}}=\frac{\partial E_{p}}{\partial Q_{x z}^{(p)}}=\frac{\partial E_{p}}{\partial \tau_{x z}^{(p t)}}=0
$$


Using eqn (13) and (14), in the first, second and third of the relations of (15) and then re-arranging and written in a combined form as below,

$$
\left[\int_{z_{p b}}^{z_{p t}}\left[\begin{array}{ccc}
\varphi_{1, z} \varphi_{1, z} & \varphi_{1, z} \varphi_{2, z} & \varphi_{1, z} \varphi_{3, z} \\
\varphi_{2, z} \varphi_{1, z} & \varphi_{2, z} \varphi_{2, z} & \varphi_{2, z} \varphi_{3, z} \\
\varphi_{3, z} \varphi_{1, z} & \varphi_{3, z} \varphi_{2, z} & \varphi_{3, z} \varphi_{3, z}
\end{array}\right] d z\right]\left\{\begin{array}{l}
\tau_{x z}^{(p b)} \\
Q_{x z}^{(p)} \\
\tau_{x z}^{(p t)}
\end{array}\right\}=-\int_{z_{p b}}^{z_{p t}}\left[\left\{\begin{array}{l}
\varphi_{1, z} \\
\varphi_{2, z} \\
\varphi_{3, z}
\end{array}\right\}\left(\sigma_{x x, x}^{(p)}+\tau_{x y, y}^{(p)}\right)\right] d z
$$

and in a compact form, as

$$
\left[k_{x z}^{p}\right]\left\{\tau_{x z}\right\}^{(p)}=\left\{f_{x z}^{(p)}\right\} .
$$

But, it can also be written as

$$
\int_{z_{p b}}^{z_{p t}}\left(\sigma_{x x, x}^{(p)} \varphi_{i, z}\right) d z=\left[\begin{array}{lll}
Q_{x x} & Q_{x y} & Q_{x s}
\end{array}\right](p)\left[\bar{H}_{p i}\right][N, x]\{\bar{\varepsilon}\}_{p}^{e} .
$$

In eqn (15), $\{\bar{\varepsilon}\}_{p}^{e}$ is the computed smoothed mid plane nodal strain vector of the $e$-th element in which the point $\mathrm{P}(x, y)$ is located, $[\mathrm{N}, x]$ is the $x$-derivative of the interpolation matrix used for calculating the strain components at $\mathrm{P}(x, y)$ from corresponding nodal components, $\mathrm{Q}_{x x}, \ldots$ etc are the relevant elements of the in plane constitutive matrix of the $p$-th ply under consideration and $\left[\bar{H}_{p i}\right]$ is given by

$$
\left[\bar{H}_{p i}\right]=\int_{z_{p b}}^{z_{p t}}\left[\left(A_{2 i}+2 A_{3 i} z\right)\left[H_{p}\right]\right] d z
$$

where $\left[H_{p}\right]$ has given in eqn (8) of Singh et al. [33].

In a similar manner as in eqn (17), one can compute,

$$
\int_{z_{p b}}^{z_{p t}}\left(\tau_{x y, y}^{(p)} \varphi_{i, z}\right) d z=\left[\begin{array}{lll}
Q_{x s} & Q_{y s} & Q_{s s}
\end{array}\right]^{(p)}\left[\bar{H}_{p i}\right][N, y]\{\bar{\varepsilon}\}_{p}^{e} .
$$

Using eqn (18) and (19), the individual terms in eqn (16) may be computed.

Now, since due to inter-laminar shear stress continuity, $\tau_{x z}^{p t}=\tau_{x z}^{(p+1) b}$, the relation in eqn (16) may be assembled for all the layers $p=1,2, \ldots N$, in a similar procedure as for the assembly of individual elements in the finite element analysis, to obtain it for the whole laminate

$$
\left[k_{x z}\right]\left\{\tau_{x z}\right\}=\left\{f_{x z}\right\}
$$

where the solution vector $\left\{\tau_{x z}\right\}$ is in the form

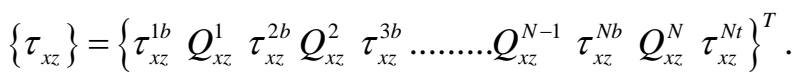


The top and bottom surface boundary conditions to be applied are given by

$$
\begin{aligned}
\tau_{x z}^{1 b} & =T_{b}(x, y) \\
\tau_{x z}^{N t} & =T_{t}(x, y)
\end{aligned}
$$

where $T_{b}(x, y)$ and $T_{t}(x, y)$ represent the applied shear traction at the bottom and top points of the laminate corresponding to the point $\mathrm{P}(x, y)$ under consideration. After applying the necessary boundary conditions, eqn (20) representing a set of linear algebraic equations can be solved to obtain directly the interlaminar stresses and the resultants in $x-z$ plane in individual plies. Following similar procedure for the transverse shear stress on the $y-z$ plane and using the second of the equilibrium equations in eqn (5),

$$
\left[k_{y z}\right]\left\{\tau_{y z}\right\}=\left\{f_{y z}\right\} \text {. }
$$

After obtaining the $\tau_{x z}$ by solving (23), the components of this vector are substituted back into eqn (10) to obtain the distribution of $\tau_{x z}^{(p)}$ in each layer $p=1, \ldots, N$. The same procedure is adopted to find the distribution of $\tau_{y z}$ through the laminate thickness.

Once the distributions of $\tau_{x z}$ and $\tau_{y z}$ have been obtained through the laminate thickness, the third of eqn (5) may be used to obtain the distribution of $\sigma_{z z}$ through the laminate thickness following similar procedures.

\section{Numerical results and discussions}

In order to demonstrate the accuracy and applicability of the proposed FE model based on RHSDT and LSE method a number of numerical examples on composites and sandwich laminates are solved. The general geometric details of the plate problem considered for different problems are shown in fig. 3 .

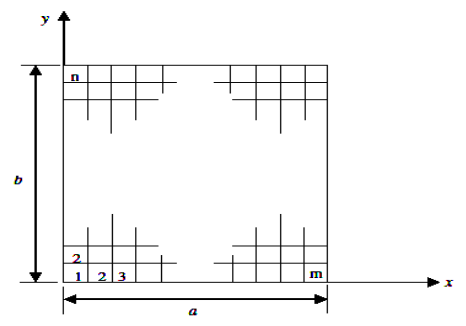

Figure 2: Rectangular plate having a mesh of $m \times n$.

\subsection{Transverse shear stress distribution through the thickness}

The proposed combined model is used for calculating the transverse shear stresses across the thickness of composites and sandwich laminates having different parametric variations and subjected to transverse static load. The 
transverse shear stresses are calculated for different problems by using the direct constitutive relationship as well as by the equilibrium equations used in the LSE method. A multiplication factor, $m=h / q_{0} a$ is used to convert the transverse shear stresses in non-dimensional forms, where $a$, is the least lateral dimension of the plate and $q_{0}$ is the amplitude of the applied sinusoidal load at the plate centre. The abbreviations 'Equil' and 'Const' preceded by an underscore in the legends of the figures indicate quantities evaluated using 3D equilibrium equations and constitutive relations, respectively.

\subsubsection{Transverse shear stress distribution in symmetric cross-ply laminate}

In this example, the problems of a three-layer [0/90/0] cross ply simply supported square laminate of equal individual thickness have been solved taking thickness ratio, $h / a=0.25$.

Although the individual layers possess different orientations but they have equal thickness and material property $\left(E_{1}=25 E_{2}, E_{2}=E_{3}, G_{12}=G_{13}=0.5 E_{2}, G_{23}=\right.$ $0.2 E_{2}, v_{12}=0.25$ and $\left.v_{13}=0.01\right)$. This is also applicable to all the subsequent problems unless mentioned otherwise.

Through-the thickness variation of non-dimensional transverse shear stress, $\tau_{y z}$, obtained at the centre of the bottom edge $(x=a / 2$ and $y=0)$ of the plate are plotted in fig. 4 plotted for the case of three layered [0/90/0] cross ply laminates.

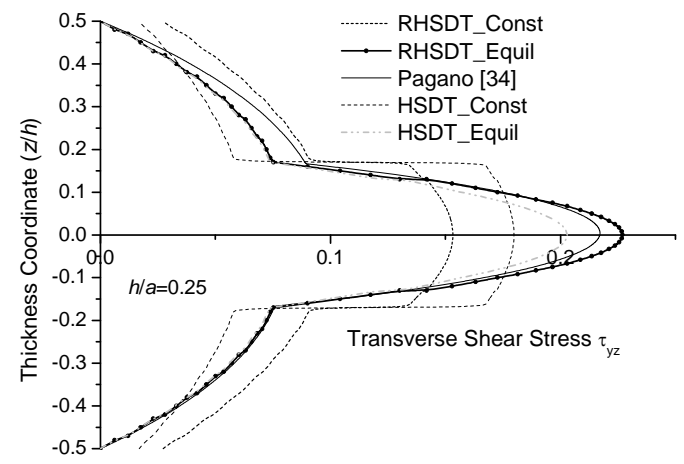

Figure 3: Variation of non-dimensional transverse shear stress $\tau_{y z}$ through the thickness of three layer [0/90/0] laminate (thickness ratio, $h / a=0.25)$.

\subsubsection{Transverse shear stress distribution in anti-symmetric cross-ply laminate}

In this example, the problem of a simply supported four-layer [0/90/0/90] square cross-ply laminate having equal layer thickness has been solved by taking thickness ratio, $h / a=0.25$. The plate is subjected to the uniform distributed loading (UDL). A multiplication factor, $m=h / q_{0} a$ is used to convert the transverse shear stresses in non-dimensional forms, where $a$, is the least lateral 


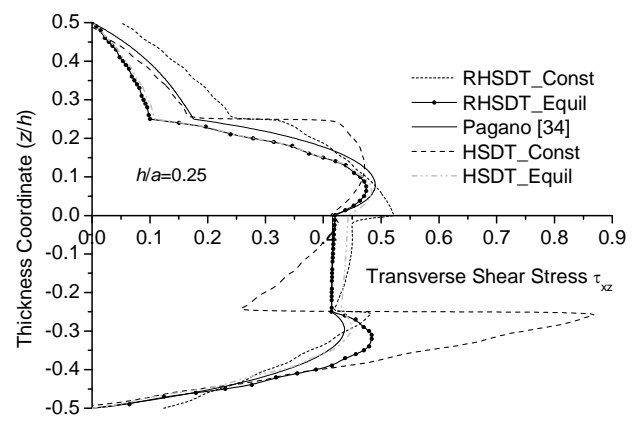

Figure 4: Variation of non-dimensional transverse shear stress $\tau_{x z}$ through the thickness of four layer [0/90/0/90] laminate (thickness ratio, $h / a=0.25)$.

dimension of the plate and $q$ is the magnitude of the applied UDL load considered at the plate centre.

\subsubsection{Transverse shear stress distribution in thick sandwich laminate}

Finally, the problem of a three layer square simply supported sandwich laminate [0/core/0] having a thickness ratio $(h / a=0.25)$ has been solved. The thickness of both the top and bottom face sheets is taken to be $h / 10$, where $h$ is the total thickness of the laminate. The plate is subjected to the sinusoidal loading. The material properties of the top and bottom face sheets of this sandwich laminate are taken same as in the previous problems and for the middle core are taken as, $E_{1}=0.04, E_{2}=0.04, E_{3}=0.5, G_{12}=0.016, G_{13}=G_{23}=0.06, v_{12}=0.25, v_{31}=0.25$ and $v_{32}=0.25$.

The results of deflections, in-plane normal stresses and transverse shear stresses obtained by using the HSDT, proposed combined model and 3D elasticity solution (Pagano [34]) are presented in table 1.

Table 1: Normalized maximum deflections and stresses for a simply supported square sandwich [0/core/0] under sinusoidal load at the plate centre.

\begin{tabular}{|l|c|c|c|c|c|c|c|}
\hline Theory & $\bar{w}$ & $\bar{\sigma}_{x x}$ & $\bar{\sigma}_{y y}$ & $\begin{array}{c}\bar{\tau}_{x z} \\
\text { Equil }\end{array}$ & $\begin{array}{c}\bar{\tau}_{x z} \\
\text { Const }\end{array}$ & $\begin{array}{c}\bar{\tau}_{y z} \\
\text { Equil }\end{array}$ & $\begin{array}{c}\bar{\tau}_{y z} \\
\text { Const }\end{array}$ \\
\hline HSDT & 4.304 & 0.982 & 0.149 & 0.2818 & 0.1410 & 0.0679 & 0.0660 \\
\hline Present & 7.637 & 1.539 & 0.253 & 0.2372 & 0.2552 & 0.1041 & 0.1169 \\
\hline Pagano [34] & 7.596 & 1.555 & 0.259 & \multicolumn{2}{|c|}{0.2386} & \multicolumn{2}{|c|}{0.1071} \\
\hline
\end{tabular}

The deflection is calculated at the midpoint of the plate $(x=a / 2, y=a / 2$ and $z=0)$ and normalized using amultiplication factor, $m=100 h^{3} E_{2} / q_{0} a^{4}$. The in plane normal stresses $\sigma_{x x}$ is calculated at $x=a / 2, y=a / 2$ and $z=h / 2$ of the plate, $\sigma_{y y}$ is calculated at $x=a / 2, y=a / 2$ and $z=-h / 2$ of the plate and both are normalized using 
the multiplication factor, $m=h^{2} / q_{0} a^{2}$. The transverse shear stresses $\tau_{x z}$ is calculated at the centre of the left edge $(x=0, y=a / 2$ and $z=0.0)$ of the plate and normalized using the multiplication factor, $m=h / q_{0} a$ while the transverse shear stresses $\tau_{y z}$ is calculated at the centre of the bottom edge $(x=a / 2, y=0$ and $z=0.0)$ of the plate and normalized using the multiplication factor, $m=h / q_{0} a$.

\section{Conclusions}

In this paper a novel numerical model has been proposed in combination of an efficient finite element (FE) model based on the refined higher order shear deformation theory (RHSDT) with a least square error (LSE) method to accurately calculate the deflections as well as stresses for different problems of composite and sandwich laminates. The proposed analysis is done in two stages. The FE model based on RHSDT is used first which calculates the deflections and in-plane stresses more accurately than any other existing $2 \mathrm{D}$ plate theory. The LSE method is then utilized to accurately predict the transverse shear stresses from the results of deflections and in-plane stresses obtained by the FE analysis based on RHSDT at the first stage of analysis. It is also interesting to note that the displacement fields and the corresponding stress fields used in the RHSDT and in the LSE method used in the proposed combined model perfectly matches with each other which is not so in case of the combination of HSDT with LSE method. Therefore, the proposed combined model may be recommended as the most efficient 2D method to accurately calculate the deflections as well as stresses (in-plane and transverse) for all types of composites and sandwich laminates.

\section{References}

[1] Yang PC, Norris $\mathrm{CH}$ and Stavsky Y., Elastic wave propagation in heterogeneous plates. Int. J. of Solids and Struct., 2, pp. 665-84, 1996.

[2] Vlachoutsis S., Shear correction factors for plates and shells. International Journal for Numerical Methods in Engineering, 33(7), pp. 1537-52, 1992.

[3] Lo KH, Christensen RM and Wu EM., A higher order theory of plate deformation, Part2. Laminated plates. Journal of Applied Mechanics Transactions of the ASME, 44, pp. 669-76, 1977.

[4] Reddy JN., A simple higher-order theory for laminated composite plates. $J$. of Applied Mechanics ASME, 51, pp. 745-52, 1984.

[5] Manjunatha B.S. and Kant T., A comparison of nine and sixteen noded quadrilateral elements based on higher order laminate theories for estimation of transverse stresses. J. of Reinf. Plas. \& Compo., 11, pp. 9861002, 1992.

[6] Reissner E., On the theory of bending of elastic plates. J. Math. Physics, 23, pp. 184-91, 1944.

[7] Shankara CA and Iyengar NGR., A $\mathrm{C}^{0}$ element for the free vibration analysis of laminated composite plates. Journal of Sound and Vibration, 191(5), pp. 721-38, 1996. 
[8] Srinivas S., A refined analysis of composite laminates. Journal of Sound and Vibration, 30, pp. 495-507, 1973.

[9] Toledano A and Murakami H., A composite plate theory for arbitrary laminate configuration. Journal of Applied Mechanics ASME, 54, pp. 18189, 1987.

[10] Robbins DH and Reddy JN., Modeling of thick composites using a layerwise laminate theory. Int. J. for Nume. Meth. in Engg., 36, pp. 655-77, 1993.

[11] Li X and Liu D., Zigzag theory for composite laminates. AIAA J., 33(6), pp. 1163-65, 1995.

[12] Di Sciuva M., A refined transverse shear deformation theory for multilayered anisotropic plates. Atti. Academia Scienze Torino, 118, pp. 279-95, 1984.

[13] Murakami H., Laminated composite plate theory with improved in-plane responses. Journal of Applied Mechanics ASME, 53, pp. 661-66, 1986.

[14] Liu D and Li X., An overall view of laminate theories based on displacement hypothesis. J. Compos. Materials, 30, pp. 1539-61, 1996.

[15] Bhaskar K and Varadan TK., Refinement of higher order laminated plate theories. AIAA J., 27, pp. 1830-31, 1989.

[16] Di Scuiva M., Multilayered anisotropic plate models with continuous interlaminar stress. Composite Structures, 22(3), pp. 149-67.

[17] Cho $M$ and Parmerter RR. An efficient higher order plate theory for laminated composites. Composite Structures, 20, pp. 113-23, 1992.

[18] Cho M and Parmerter RR., Efficient higher order composite plate theory for general lamination configurations. AIAA J, 31(7), pp. 1299-1306, 1993.

[19] Carrera E., On the use of the Murakami's zig-zag function in the modeling of layered plates and shells. Comp. and Structures, 82, pp. 541-54, 2004.

[20] Demasi L., Refined multilayered plate elements based on Murakami zigzag functions. Composite Structures, 70, pp. 308-16, 2005.

[21] Murakami H., Laminated composite plate theory with improved in-plane response. Journal of Applied Mechanics ASME, 53, pp. 661-6, 1986.

[22] Demasi L., Mixed plate theories based on generalized unified formulation Part IV: zig-zag theories. Composite Structures, 87, pp. 195-205, 2009.

[23] Demasi L., Mixed plate theories based on generalized unified formulation. Part V: Results. Composite Structures, 88, pp. 1-16, 2004.

[24] Kapuria S, Dumir PC and Ahmed A., Efficient coupled zigzag theory for hybrid piezoelectric beams for thermoelectric load. AIAA J., 42, pp. 38394, 2004.

[25] Kapuria S and Achary GGS., A coupled zigzag theory for the dynamics of piezoelectric hybrid cross-ply plates. Arch. Appl. Mech., 75, pp. 42-57, 2005.

[26] Zhen W and Wanji C., A C0-type higher-order-theory for bending analysis of laminated composite and sandwich plates. Composite Structures, 92, pp. 653-61, 2010. 
[27] Zhen W, Wanji C and Xiaohui R., An accurate higher-order theory and $\mathrm{C}^{0}$ finite element for free vibration analysis of laminated composite and sandwich plates. Composite Structures, 92, pp. 1299-1307, 2010.

[28] Bhar A and Satsangi SK., Accurate transverse stress evaluation in composite/sandwich thick laminates using $\mathrm{C}^{0}$ HSDT and a novel postprocessing technique. Eur. J. of Mechanics and Solids, 30, pp. 46-53, 2011.

[29] Cook Robert D, Malkus David S, Michael Plesha E and Witt Robert J., Concepts and Applications of Finite Element Analysis, $4^{\text {th }}$ Edition John Wiley \& Sons Pvt. Ltd. , 2003.

[30] Chakrabarti A and Sheikh AH., A new plate bending element based on higher order shear deformation theory for the analysis of composite plates. Finite Elements in Analysis and Design, 39(9), pp. 883-903, 2003.

[31] Chakrabarti A and Sheikh AH., Analysis of laminated sandwich plates based on interlaminar shear stress continuous plate theory. Journal of Engineering Mechanics ASCE, 131(4), pp. 377-84, 2005.

[32] Pandit MK, Sheikh AH and Singh BN., An improved higher order zigzag theory for the static analysis of laminated sandwich plate with soft core. Finite Elements in Analysis and Design, 44, pp. 602-10, 2008.

[33] Singh SK, Chakrabarti A, Bera P and JSD Sony., An efficient $C^{0}$ FE model for the analysis of composites and sandwich laminates with general layup. Latin American Journal of solids and structures, 8, pp. 197-212, 2011.

[34] Pagano NJ., Exact solution for rectangular bidirectional composites and sandwich plates. Journal of Composite Materials, 4, pp. 20-34, 1970. 